Results Average MAFA-Ratio values calculated from pre- and post-stent placement were significantly lower after deployment of the 64-wire device $($ mean $=0.62 \pm 0.09$ ) compared to the 48 -wire device $(0.71 \pm 0.06) ; \mathrm{p}=<0.05$.

Conclusions Our in-vitro results show that the 64 wire FDS (Evolve) had superior flow diversion effect compared to the 48 wire FDS (Pipeline), suggesting that 64-wires are superior to 48-wire designs for flow diversion efficacy.

Disclosures N. Cancelliere: None. P. Nicholson: None. K. Mendes: None. E. Orru: None. T. Krings: None. V. Mendes Pereira: 1; C; Philips Healthcare. 2; C; Stryker, Medtronic.

\section{P-031 PRELIMINARY ASSESSMENT OF THE PPODA-QT LLIQUID EMBOLIC IN A RABBIT ELASTASE ANEURYSM MODEL}

${ }^{1} \mathrm{~T}$ Becker* ${ }^{*}{ }^{2} \mathrm{~A}$ Huckleberry, ${ }^{1} \mathrm{~W}$ Merritt, ${ }^{3} \mathrm{~T}$ Cotter, ${ }^{1} \mathrm{C}$ Settanni, ${ }^{4} \mathrm{~A}$ Ducruet. ${ }^{1}$ Bioengineering Devices Lab, Mechanical Engineering, Northern Arizona University, Flagstaff, $A Z_{i}$ ${ }^{2}$ Bioengineering Devices Lab, Biology, Northern Arizona University, Flagstaff, $A Z$; ${ }^{3}$ Bioengineering Devices Lab, Physics, Northern Arizona University, Flagstaff, AZ; ${ }^{4}$ Barrow Brain and Spine, Barrow Neurological Institute, Phoenix, AZ

10.1136/neurintsurg-2019-SNIS.67

Introduction PPODA-QT is a novel liquid embolic under development for the treatment of cerebral aneurysms. The material is prepared and undergoes gelation in a predictable manner over a pre-set length of time for controllable delivery to the aneurysm with balloon protection. We utilized a rabbitelastase aneurysm model with histological analysis to evaluate tissue response and biocompatibility following PPODA-QT embolization.

Materials and Methods Experimental elastase-induced aneurysms were created via endovascular technique in New Zealand White Rabbits by incubating $100 \mathrm{U}$ of elastase, mixed 1:1 with and $0.5 \mathrm{M}$ calcium chloride, within the carotid stump for 20 minutes with Fogarty balloon protection. Three weeks after elastase treatment, the aneurysms were embolized using balloon remodeling with a Scepter XC (Microvention) across the aneurysm neck and PPODA-QT was delivered behind the balloon with a Velocity microcatheter (Penumbra). Rabbit control and aneurysm tissues were harvested at acute, 1-month, and 3-month timepoints. All tissues were prepared for histology assessment with Van Gieson and H\&E staining protocols. Results The rabbit-elastase aneurysms developed into small aneurysms ( $<10 \mathrm{~mm}$ dome height) with highly variable neck morphologies and beyond-wide dome-to-neck $(d: n)$ ratios. Histological data captured areas of direct device contact with the aneurysm wall and neck, demonstrating elastin reorganization of vessel wall into a smooth muscle layer after 1-month survivals. At the aneurysm neck, a homogenous neointimal (NI) tissue layer $(200-300 \mu \mathrm{m})$ formed at the PPODA-QT interface, sealing off the parent vessel from the aneurysm dome (figure 1). Results reveal no symptoms of acute inflammation from the elastase incubation and no adverse immune response was evident at 1- and 3-month survival timepoints following PPODA-QT embolization.

Conclusion Following PPODA-QT embolization, NI tissue growth and remodeling was noted with minimal immunological response, indicating that PPODA-QT can be successfully delivered to wider-neck aneurysms and promote aneurysm tissue reorganization and stabilization that facilitates continuous healing at the aneurysm neck. Because experimental aneurysms were uniformly small with inconsistent neck morphology, further testing in larger aneurysm models (i.e. canines) is planned to optimize device delivery and verify healing responses prior to human clinical investigation.

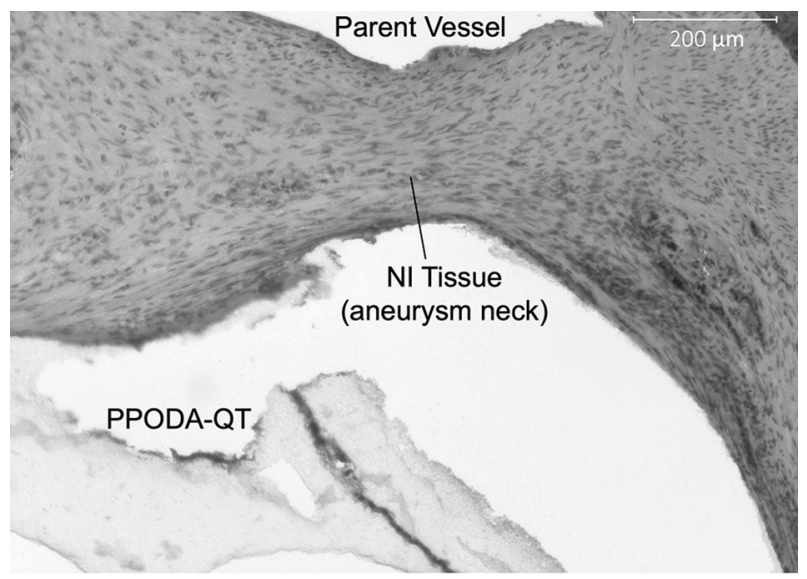

Abstract P-031 Figure 1 Continuous neo-intimal (NI) tissue growth across the neck of a rabbit elastase aneurysm survived 3 months, separating the parent vessel from the PPODA-QT implant

Disclosures T. Becker: 1; C; Brain Aneurysm Foundation Grant-2018. 4; C; Aneuvas Technologies, Inc.. 5; C; Northern Arizona University, Aneuvas Technologies, Inc. A. Huckleberry: 1; C; Brain Aneurysm Foundation Grant-2018. W. Merritt: 1; C; Brain Aneurysm Foundation Grant-2018. 5; C; Northern Arizona University. T. Cotter: 1; C; Brain Aneurysm Foundation Grant-2018. 5; C; McGill University. C. Settanni: 1; C; Brain Aneurysm Foundation Grant-2018. 5; C; Northern Arizona University. A. Ducruet: 1; C; Brain Aneurysm Foundation Grant-2018. 5; C; Barrow Neurological Institute.

\section{P-032 POST PROCEDURAL LOW DOSE HEPARIN DRIP REDUCES THROMBOTIC EVENTS FOLLOWING PIPELINE EMBOLIZATION, WITHOUT INCREASING HEMORRHAGIC COMPLICATIONS}

I Rybkin, J Cooper, G Kaur, F Al-Mufti, C Gandhi, J Santarelli*. Westchester Medical Center, Valhalla, NY

\subsection{6/neurintsurg-2019-SNIS.68}

Purpose Pipeline embolization devices (PEDs) are associated with inherent thrombotic complications as well as potentially catastrophic spontaneous intraparenchymal hemorrhage. Antiplatelet and anticoagulation therapy must be optimized to reduce thrombotic complications without increasing the incidence of hemorrhage. Post-procedural low dose heparin drip is a prophylactic measure to reduce ischemic stroke risk, with no published data to date reporting efficacy and complications. Here we report the thrombotic and hemorrhagic complication rates of patients receiving post-operative low dose heparin drip, and we compare our results to the published literature rates of patients not receiving such prophylaxis.

Materials and methods We completed a retrospective review of patients who had intracranial aneurysms treated with the Pipeline Embolization Device at Westchester Medical Center. A total of 73 individuals received post-operative low dose heparin for an average of 18 hours. Thrombotic and hemorrhagic complications were identified and reported. These rates were 